\title{
The Dirichlet problem for semilinear elliptic equations in an infinite sector
}

\author{
A. El Hajj ${ }^{1} \cdot$ H. Ibrahim²
}

Received: 10 October 2017 / Accepted: 2 February 2019 / Published online: 14 May 2019 (c) Fondazione Annali di Matematica Pura ed Applicata and Springer-Verlag GmbH Germany, part of Springer Nature 2019

\begin{abstract}
We consider the Dirichlet problem for a semilinear elliptic equation in an unbounded sectorial domain $\Omega$ of the two-dimensional space. The problem is supplemented with a limiting behavior related to a prescribed root $z$ of the nonlinearity of the equation. The one-dimensional setting of the problem has a unique solution $V_{z}$, and the goal of the present paper is to construct a two-dimensional positive and bounded solution $u$ approaching $V_{z}(d)$ when $d=d(x, \partial \Omega) \rightarrow \infty$. This is established using sub- and supersolutions method and employing a sliding argument.
\end{abstract}

Keywords Semilinear elliptic equations $\cdot$ Asymptotic behavior $\cdot$ Sliding method

Mathematics Subject Classification 35J61 · 35B40

\section{Introduction and main result}

While studying the properties of particular solutions of PDEs using some blow-up arguments, we are naturally lead to solving PDEs in unbounded domains such as half-spaces. As an example, we refer to [1] where the study of the boundary layer of the solution $u_{\varepsilon}$ of the following problem

$$
\left\{\begin{array}{l}
\varepsilon^{2} \Delta u_{\varepsilon}+f\left(x, u_{\varepsilon}\right)=0, \\
u_{\varepsilon}=0 \text { on the boundary }
\end{array}\right.
$$

leads to the study of an elliptic PDE in the half-space.

Positive solutions of the semilinear elliptic equation

$$
\Delta u+f(u)=0
$$

enjoy symmetry and monotonicity properties on symmetric domains. This is directly related to the symmetry of the operator $\Delta$. For this reason, the above equation has been widely studied

\footnotetext{
$凶 \quad$ A. El Hajj

elhajjah@utc.fr

1 Université de Technologie de Compiègne, LMAC, 60205 Compiègne Cedex, France

2 Mathematics Department, Faculty of Sciences (I), Lebanese University, Hadath, Lebanon
} 
on domains like balls, half-spaces or cylindrical unbounded domains. For the case of bounded domains, we refer the reader to [5,11] and, for the case of unbounded domains, we refer to $[2,3,6,7]$. These articles have been instrumental in almost all results on monotonicity and symmetry of the solution of (1.1). They have also been crucial in investigating the properties of bounded solutions by relying mainly on the maximum principle and the moving plane method or the sliding method. Indeed, for bounded solutions on unbounded domains, these methods may also be used to study the limiting profile and its relation with the zeros of the nonlinearity $f$ (see for instance [8,9,12]). In fact, it has been shown in [9] that, under particular assumptions on $f$, any positive and bounded solution of (1.1) in the quarter-plane converges asymptotically to a one-dimensional limiting profile. This result has been obtained using two different approaches: PDEs and infinite-dimensional dynamical systems.

Motivated by the asymptotic behavior of solutions of (1.1) on unbounded domains, we show how to construct a particular positive and bounded solution on an infinite sectorial domain

$$
\Omega=\left\{(r \cos \theta, r \sin \theta) \in \mathbb{R}^{2} ; r>0,|\theta|<\theta_{0} \leq \pi / 2\right\},
$$

converging to a prescribed root $z$ of $f$. More precisely, we are interested in the existence of the Dirichlet problem

$$
\begin{cases}\Delta u+f(u)=0 & \text { in } \quad \Omega \\ u=0 & \text { on } \quad \partial \Omega,\end{cases}
$$

where $f:\left[0, \infty\left[\rightarrow \mathbb{R}\right.\right.$ is a locally Lipschitz continuous function. If $\theta_{0}=\frac{\pi}{2}$, then $\Omega$ becomes the half-plane $\left\{\left(x_{1}, x_{2}\right) \in \mathbb{R}^{2} ; x_{1}>0\right\}$ and a bounded positive solution of (1.2) formally converges, as $x_{1} \rightarrow \infty$, to a positive root $z$ of $f$. In fact, under additional assumptions on $f$, it turns out that the solution of (1.2) is a function of $x_{1}$ alone and it can, therefore, be determined by solving an ODE (see for instance [4]).

In this paper, we want to look at the problem from a different point of view. By fixing a suitable root $z>0$ of $f$, we first consider the following ODE that could be considered as the one-dimensional setting of our problem:

$$
\left\{\begin{array}{l}
V^{\prime \prime}+f(V)=0 \text { in }(0, \infty), \\
V(0)=0<V(t)<z=V(\infty) \text { for all } t>0, \\
V^{\prime}(t)>0 \text { for all } t \geq 0 .
\end{array}\right.
$$

This ODE has a unique regular solution

$$
V_{z} \in C^{2}\left(\mathbb{R}_{+}\right)
$$

and, although it is a direct result, we will show it in Proposition 3.1 for the sake of completeness. We aim to construct a solution of (1.2) satisfying

$$
0<u \leq V_{z}(d) \text { in } \Omega \text { and }\left(V_{z}(d)-u\right) \rightarrow 0 \text { as } d \rightarrow \infty,
$$

where

$$
d=d(x)=d(x, \partial \Omega)
$$

is the distance from $x$ to the boundary of $\Omega$. The result follows by showing that $\bar{u}=V_{z}(d)$ is a supersolution of (1.2) and by finding a suitable subsolution $\underline{u}$ of (1.2) with

$$
0 \leq \underline{u} \leq V_{z}(d) \text { and } \underline{u} \rightarrow z \text { as } d \rightarrow \infty .
$$

We then use the fact that $V_{z}(d) \rightarrow z$ as $d \rightarrow \infty$ and we employ the method of sub- and supersolutions for semilinear elliptic equations. 
Remark 1.1 We note that the limit in (1.4) could also be obtained by taking $\bar{u}=z$ as supersolution. However, our interest is to construct a solution that is bounded from above by $V_{z}$ which is better upper bound than the one given by $z$.

We now turn to the exact assumptions on $f$ and $z$. Assume that $f$ satisfies:

$$
f(0)=0 \text { and } \liminf _{t \rightarrow 0^{+}} \frac{f(t)}{t}>0 .
$$

Note that if $f$ is differentiable at 0 then $f^{\prime}(0)>0$. Throughout all this paper, the function $F$ denotes a primitive of $f$ :

$$
F(s)=\int_{0}^{s} f(t) \mathrm{d} t .
$$

Let $\mathcal{Z}=\{z \geq 0 ; f(z)=0\}$ be the set of all nonnegative roots of $f$ and let $\mathcal{Z}^{+} \subseteq \mathcal{Z} \backslash\{0\}$ be the set defined by:

$$
\mathcal{Z}^{+}=\{z \in \mathcal{Z} \backslash\{0\} ; F(s)<F(z) \text { for all } 0<s<z\},
$$

which is assumed to be nonempty $\mathcal{Z}^{+} \neq \emptyset$. The following remark is useful.

Remark 1.2 Using (1.6), we note that if $z \in \mathcal{Z}^{+}$then $F(z)>0$.

We are now ready to state our main theorem.

Theorem 1.3 We assume that $\mathcal{Z}^{+} \neq \emptyset$. Let $f$ satisfy (1.6) and let $z \in \mathcal{Z}^{+}$such that

$$
\exists \delta>0 \text { such that } f \geq 0 \text { on }(z-\delta, z) .
$$

Then, there exists a solution $u$ of (1.2) satisfying (1.4).

Remark 1.4 Since $V_{z}$ is a solution of (1.3) and as a consequence of (1.4), we directly get

$$
u \rightarrow z \text { as } d \rightarrow \infty \text {. }
$$

The existence result of Theorem 1.3 is based on sub- and supersolution method by carefully constructing a sub- and a supersolution that will be denoted $\underline{u}$ and $\bar{u}$, respectively. Those solutions should be understood to satisfy the equation in a weak sense, i.e.,

$$
\left\{\begin{array}{l}
\underline{u}, \bar{u} \in C(\bar{\Omega}), \quad \underline{u}, \bar{u}=0 \text { on } \partial \Omega, \\
\int_{\Omega} \underline{u} \Delta \varphi+f(\underline{u}) \varphi \geq 0, \quad \forall \varphi \in C_{0}^{\infty}(\Omega), \varphi \geq 0, \\
\int_{\Omega} \bar{u} \Delta \varphi+f(\bar{u}) \varphi \leq 0, \quad \forall \varphi \in C_{0}^{\infty}(\Omega), \varphi \geq 0 .
\end{array}\right.
$$

If $\underline{u} \leq \bar{u}$, we can apply the sub- and supersolution method (see for instance [10,13]) and infer the existence of a solution $u$ of (1.2) such that $\underline{u} \leq u \leq \bar{u}$. This solution is understood to be continuous on $\bar{\Omega}$ and to satisfy the equation in a weak sense as described above. Moreover, from standard elliptic estimates, the solution is of class $C^{2}(\Omega)$. The fact of getting a classical solution starting from weak sub- and supersolutions can also be reached by adapting [10, Theorem 3] to the case of unbounded domains with boundary. This idea is already used in [13, Theorem 4.1].

The remaining part of this paper will be organized as follows. In Sect. 2, we show how to construct an approximate subsolution $\underline{u}_{\varepsilon}$ using variational arguments for elliptic equations. However, the determination of the subsolution $\underline{u}$ will be postponed to Sect. 4 . In Sect. 3, we find a supersolution $\bar{u}$ given by the solution $V_{z}$ of (1.3). Later, in Sect. 4, we use a sliding argument to compare $\underline{u}$ and $\bar{u}$ and finally present the proof of Theorem 1.3. 


\section{Construction of an approximate subsolution $\underline{u}_{\varepsilon}$}

As a first step to find a subsolution $\underline{u}$ satisfying (1.5), we aim to construct an approximate subsolution of (1.2) taking a constant value (sufficiently close to $z$ ) on the set

$$
\Omega_{R}=\{x \in \Omega: d(x)>R\} .
$$

In particular, we prove

Proposition 2.1 Assume that $f$ satisfies (1.6) and let $z \in \mathcal{Z}^{+}$satisfying (1.7). Then, for every $\varepsilon>0$ small enough, there exists $R=R(\varepsilon)>0$ and a subsolution $\underline{u}_{\varepsilon}$ of (1.2) such that:

$$
0<z-\underline{u}_{\varepsilon}<\varepsilon \text { on } \Omega_{R} .
$$

The proof is based on finding suitable radial solutions of the following Dirichlet problem on sufficiently large balls $B_{R}=B_{R}(0) \subseteq \mathbb{R}^{2}$ :

$$
\begin{cases}\Delta w+f(w)=0 & \text { in } \overline{B_{R}} \\ 0 \leq w<z & \text { in } \overline{B_{R}} \\ w=0 & \text { on } \partial B_{R} \\ w(0)=\frac{\max }{B_{R}} w>z-\varepsilon . & \end{cases}
$$

Since the first equation of (2.10) does not explicitly depend on the variables $x_{1}$ and $x_{2}$, a translation of the solution in any direction is again a solution of (2.10) on the resulting domain. The idea then is to move the ball completely inside the sectorial domain $\Omega$ and extend the solution by zero, thus obtaining a subsolution of (1.2). By allowing the ball to be displaced in all of $\Omega$, we are lead to a family of subsolutions whose supremum is $\underline{u}_{\varepsilon}$; and we obtain $\Omega_{R}$ as the set of all centers of $B_{R}$ (see Fig. 1). We now move to the details. Our next lemma deals with the existence of radial solutions on large balls when $z=z_{1}$ is the first positive root. The only reason to write this result independently is to show that, in this case, the arguments leading to the existence are almost immediate.
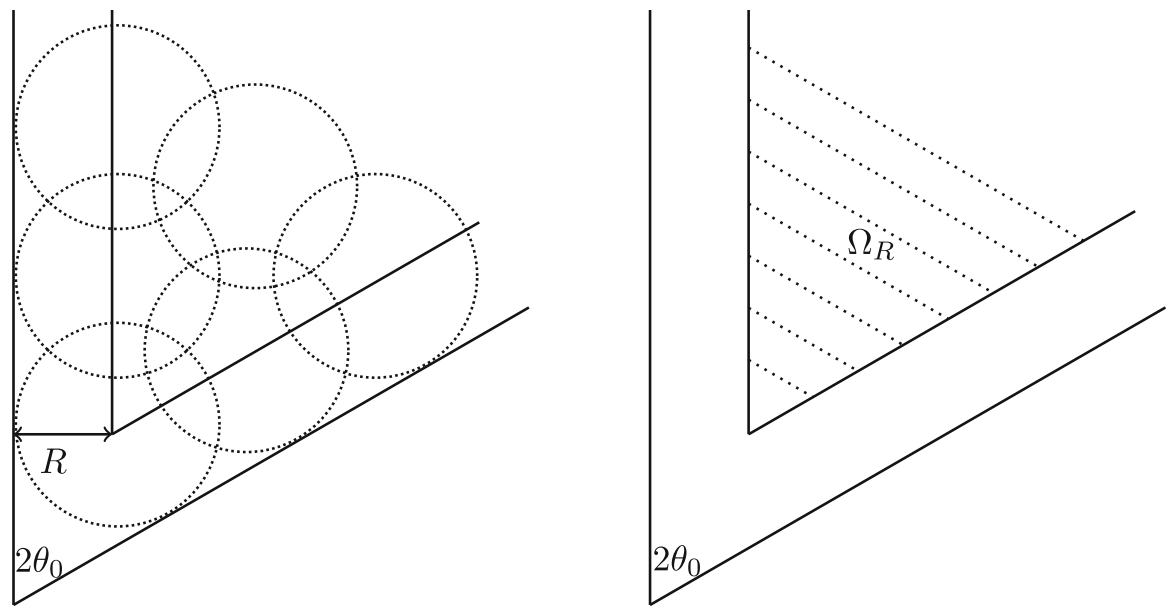

Fig. 1 Geometric interpretation of the set $\Omega_{R}$ 
Lemma 2.2 Let $z_{1}>0$ be the first positive root of $f$. For every $\varepsilon \in\left(0, z_{1}\right)$, there exists $R=R(\varepsilon)>0$ and a classical subsolution $u_{\varepsilon, R}$ of (2.10) with $z=z_{1}$.

Proof We consider the Dirichlet eigenvalue problem for the Laplace operator on $B_{R}$ :

$$
\begin{cases}\Delta \phi_{R}+\lambda_{R} \phi_{R}=0 & \text { in } B_{R}, \\ \phi_{R}>0 & \text { on } B_{R}, \\ \phi_{R}=0 & \text { on } \partial B_{R}, \\ \left\|\phi_{R}\right\|_{L^{\infty}\left(B_{R}\right)}=\phi_{R}(0)=1 . & \end{cases}
$$

The existence and uniqueness of a positive regular eigenfunction of this problem are classical. It is also standard that $\lambda_{R} \rightarrow 0$ as $R \rightarrow \infty$. Fix some $a \in\left(z_{1}-\varepsilon, z_{1}\right)$. Since $f>0$ on the interval ]0, $a$ ] and satisfies (1.6) then

$$
k:=\inf _{t \in[0, a]} \frac{f(t)}{t}>0 .
$$

As $\lambda_{R} \rightarrow 0$ when $R$ increases, we may find $\lambda_{R}$ small enough so that

$$
\lambda_{R} \leq k .
$$

Direct computations lead

$$
f(t) \geq \lambda_{R} t \text { for all } 0 \leq t \leq a .
$$

Setting $u_{\varepsilon, R}=a \phi_{R}$, we obtain the required solution.

In order to find $\underline{u}_{\varepsilon, R}$ for $z \neq z_{1}$, the tools used in Lemma 2.2 are no longer valid as $f$ may encounter negative values contradicting (2.11) even for large $R$. We rather make use of variational arguments for semilinear elliptic equations as well as the condition (1.7).

Lemma 2.3 Let $z \in \mathcal{Z}^{+}$be arbitrary. Assume (1.7) holds true. For every $\varepsilon>0$ small enough, there exists $R=R(\varepsilon)>0$ and a classical solution $u_{\varepsilon, R}$ of (2.10).

Proof The proof is divided into three steps.

Step 1. The choice of $\varepsilon$ From (1.7), we know that $f \geq 0$ on $(z-\delta, z)$ for some $\delta>0$. Let $\tilde{f}$ be the function defined over $\mathbb{R}$ by:

$$
\tilde{f}(t)= \begin{cases}f(t) & \text { if } 0 \leq t \leq z, \\ 0 & \text { elsewhere }\end{cases}
$$

and set

$$
\widetilde{F}(s)=\int_{s}^{z} \tilde{f}(t) \mathrm{d} t
$$

Using the above arguments and the fact that $z \in \mathcal{Z}^{+}$with Remark 1.2, we get:

$$
\left\{\begin{array}{l}
\widetilde{F}>0 \text { on }(-\infty, z), \\
\widetilde{F}=0 \text { on }[z, \infty), \\
\widetilde{F} \text { is decreasing on }(z-\delta, z) .
\end{array}\right.
$$

The function $\widetilde{F}$ being continuous and positive on the compact $[0, z-\delta]$ ensures the existence of $\alpha>0$ such that $\widetilde{F}>\alpha$ on $[0, z-\delta]$. By the continuity of $\widetilde{F}$ at $z$ and as $\widetilde{F}(z)=0$, we may find a small $0<\delta^{\prime}<z$ such that

$$
0<\widetilde{F}<\alpha \text { on }\left(z-\delta^{\prime}, z\right)
$$


We then choose any $\varepsilon>0$ such that

$$
0<\varepsilon<\min \left\{\delta, \delta^{\prime}\right\} .
$$

Step 2. Construction of a subsolution First note that the function $\widetilde{F}$ is nonnegative and Lipschitz on $\mathbb{R}$. Let $r$ be any positive real number. Define

$$
\mathcal{E}_{r}(v)=\frac{1}{2} \int_{B_{r}}|\nabla v|^{2}+\int_{B_{r}} \widetilde{F}(v)
$$

for all $v \in H_{0}^{1}\left(B_{r}\right)$. This functional $\mathcal{E}_{r}$ is well defined in $H_{0}^{1}\left(B_{r}\right)$ and, from Poincaré inequality and the nonnegativity of $\widetilde{F}$, it is coercive. Therefore, by Rellich's and Lebesgue's theorems, it has a minimum $v_{r}$ in $H_{0}^{1}\left(B_{r}\right)$. The function $v_{r}$ is a weak and hence, from the elliptic regularity theory, a classical $C^{2}\left(\overline{B_{r}}\right)$ solution of the equation

$$
\begin{cases}\Delta v_{r}+\tilde{f}\left(v_{r}\right)=0 & \text { in } B_{r}, \\ v_{r}=0 & \text { on } \partial B_{r} .\end{cases}
$$

Since $\tilde{f}=0$ on $(-\infty, 0] \cup[z, \infty)$, it follows from the strong maximum principle that $0 \leq v_{r} \leq z$ in $\overline{B_{r}}$, consequently $\widetilde{f}\left(v_{r}\right)=f\left(v_{r}\right)$ in $\overline{B_{r}}$ and therefore $v_{r}$ is a solution of

$$
\begin{cases}\Delta v_{r}+f\left(v_{r}\right)=0 & \text { in } B_{r}, \\ v_{r}=0 & \text { on } \partial B_{r} .\end{cases}
$$

Again, the strong maximum principle and the fact that $v_{r}=0$ on $\partial B_{r}$ ensure $v_{r}<z$ in $\overline{B_{r}}$. It also follows from [11] that the solution $v_{r}$ is radially symmetric $v_{r}(x)=v_{r}(|x|)$ and decreases with respect to $|x|$ if $v_{r} \not \equiv 0$, hence attaining its maximum at the center 0 . As a conclusion, one gets:

$$
0 \leq v_{r}(x) \leq v_{r}(0)=\max _{\overline{B_{r}}} v_{r}<z \text { in } \overline{B_{r}} .
$$

Step 3. A suitable subsolution We first show that, for the above choice (2.13) of $\varepsilon$, the following estimate holds

$$
\widetilde{F}(s) \geq \widetilde{F}(z-\varepsilon) \text { for every } 0 \leq s \leq z-\varepsilon .
$$

In fact, if $0 \leq s \leq z-\delta$ then $\widetilde{F}(s) \geq \alpha \geq \widetilde{F}(z-\varepsilon)$. If $z-\delta \leq s \leq z-\varepsilon$ then, as $\widetilde{F}$ decreases, we also get $\widetilde{F}(s) \geq \widetilde{F}(z-\varepsilon)$.

To complete the proof, it suffices to show that, for $\varepsilon$ given by (2.13), there exists $r>0$ such that $v_{r}(0)>z-\varepsilon$. Assume otherwise that $v_{r} \leq z-\varepsilon$ for all $r>0$. By (2.14) and the positivity of $\widetilde{F}$, we obtain:

$$
\mathcal{E}_{r}\left(v_{r}\right) \geq \int_{B_{r}} \widetilde{F}\left(v_{r}\right) \geq \int_{B_{r}} \widetilde{F}(z-\varepsilon)=\pi \widetilde{F}(z-\varepsilon) r^{2}, \quad \forall r>0 .
$$

On the other hand, for $r>1$, define the test function $w_{r} \in H_{0}^{1}\left(B_{r}\right)$ by:

$$
w_{r}(x)= \begin{cases}z & \text { for }|x|<r-1, \\ z(r-|x|) & \text { for } \quad r-1 \leq|x| \leq r .\end{cases}
$$

As $w_{r} \equiv z$ for $|x|<r-1$ and since $\widetilde{F}(z)=0$, we deduce that $\left|\nabla w_{r}\right|^{2}$ and $\widetilde{F}\left(w_{r}\right)$ are supported on the annulus $\{r-1 \leq|x| \leq r\}$. Thus, for some constant $C$ independent of $r$, we get:

$$
\mathcal{E}_{r}\left(w_{r}\right) \leq C(2 r-1), \quad \forall r>1 .
$$


But since $v_{r}$ is the minimizer of $\mathcal{E}_{r}$, we have $\mathcal{E}_{r}\left(v_{r}\right) \leq \mathcal{E}_{r}\left(w_{r}\right)$ and thus

$$
\widetilde{F}(z-\varepsilon) r^{2} \leq \frac{C}{\pi}(2 r-1), \quad \forall r>1 .
$$

By noticing that $\widetilde{F}(z-\varepsilon)>0$, we may deduce that the above inequality does not hold for large $r$. Therefore, there exists $R>0$ such that $v_{R}(0)>z-\varepsilon$. Finally, set $u_{\varepsilon, R}=v_{R}$ and the proof of the lemma is complete.

We now turn to the existence of subsolutions of (1.1) on the whole domain $\mathbb{R}^{2}$. This is an immediate consequence of the above lemmas. Precisely, we have

Corollary 2.4 Let $u_{\varepsilon, R}$ be the subsolution obtained in Lemma 2.2 or the solution obtained in Lemma 2.3. We set

$$
\underline{u}_{\varepsilon, R}=\left\{\begin{array}{l}
u_{\varepsilon, R} \text { in } \overline{B_{R}}, \\
0 \quad \text { otherwise. }
\end{array}\right.
$$

Then $\underline{u}_{\varepsilon, R}$ is a weak subsolution of (1.1) in $\mathbb{R}^{2}$.

Proof Let $\varphi \in C_{0}^{\infty}\left(\mathbb{R}^{2}\right), \varphi \geq 0$. We set

$$
\mathcal{O}=B_{R} \cap \operatorname{supp}(\varphi),
$$

then, integrating by parts, we get

$$
\int_{\mathbb{R}^{2}} \underline{u}_{\varepsilon, R} \Delta \varphi+f\left(\underline{u}_{\varepsilon, R}\right) \varphi=\int_{\mathcal{O}} \underline{u}_{\varepsilon, R} \Delta \varphi+f\left(\underline{u}_{\varepsilon, R}\right) \varphi \geq-\int_{\partial B_{R} \bigcap \operatorname{supp}(\varphi)} \varphi \frac{\partial u_{\varepsilon, R}}{\partial v},
$$

where $v$ is the outward normal to the boundary $\partial B_{R} \cap \operatorname{supp}(\varphi)$. We note that $\frac{\partial u_{\varepsilon, R}}{\partial v}<0$ on $\partial B_{R}$ by the Hopf Lemma and therefore $\int_{\mathbb{R}^{2}} \underline{u}_{\varepsilon, R} \Delta \varphi+f\left(\underline{u}_{\varepsilon, R}\right) \varphi \geq 0$.

We now present the

Proof of Proposition 2.1 For $\varepsilon$ satisfying (2.13) and $R=R(\varepsilon)>0$ given by Lemma 2.3, let $\underline{u}_{\varepsilon, R}$ be the subsolution obtained by Corollary 2.4. Let $\Omega_{R}$ be as in Theorem 1.3 and fix $y \in \Omega_{R}$.

Consider $\underline{u}_{\varepsilon, R}^{y}$; the translation of $\underline{u}_{\varepsilon, R}$ by the vector $y$,

$$
\underline{u}_{\varepsilon, R}^{y}(x)=\underline{u}_{\varepsilon, R}(x-y) .
$$

Since the space variable $x$ appears only through the solution $u$ in equation (1.1), it directly follows that $\underline{u}_{\varepsilon, R}^{y}(x)$ is a subsolution of (1.2). In order to obtain a subsolution satisfying (2.9), we take

$$
\underline{u}_{\varepsilon}=\left.\sup _{y \in \Omega_{R}} \underline{u}_{\varepsilon, R}^{y}\right|_{\Omega},
$$

which is also (see for instance [10,13,14]) a subsolution of (1.2). Here, one can easily check that $\underline{u}_{\varepsilon, R}^{y}<z$ hence $\underline{u}_{\varepsilon} \leq z$ and then $\underline{u}_{\varepsilon}<z$ from the strong maximum principle. Also, we notice that $\underline{u}_{\varepsilon}$ is constant on $\Omega_{R}$ for

$$
\underline{u}_{\varepsilon}(x)=\underline{u}_{\varepsilon, R}^{x}(x)=\underline{u}_{\varepsilon, R}(0)=\max _{\overline{B_{R}}} \underline{u}_{\varepsilon, R}, \quad \forall x \in \Omega_{R},
$$

therefore

$$
z-\underline{u}_{\varepsilon}(x)<\varepsilon, \quad \forall x \in \Omega_{R},
$$

and this ends the proof. 


\section{Construction of a supersolution $\bar{u}$}

In this section, we construct a supersolution of (1.2) by relying on the solution $V_{z}$ of the ODE (1.3). We prove

Proposition 3.1 Let $z \in \mathcal{Z}^{+}$, then there exists a unique regular solution $V_{z}$ of (1.3). Moreover, the function

$$
\bar{u}=V_{z}(d)
$$

is a supersolution of (1.2).

Proof The existence of $V_{z}$ is classical and uses standard shooting arguments. However, we present the proof for the sake of completeness. In light of Remark 1.2, we know that $F(z)>0$, and therefore, we consider the following problem

$$
\left\{\begin{array}{l}
V^{\prime \prime}+f(V)=0 \text { in }(0, \infty), \\
V(0)=0, \quad V^{\prime}(0)=\sqrt{2 F(z)}>0,
\end{array}\right.
$$

where existence and uniqueness on a maximal interval $[0, b) \subseteq \mathbb{R}$ follow by applying a fixed point argument. Multiplying the first equation of (3.17) by $V^{\prime}$, and thanks to the initial data in (3.17), and the fact that $F^{\prime}=f$, we obtain

$$
\frac{1}{2}\left(V^{\prime}(t)\right)^{2}+F(V(t))=F(z), \forall t \in[0, b) .
$$

Since $f(z)=0$, easy computations show that the constant function $g(t)=z$ is a solution of the first equation of (3.17). The function $V$ can not touch $z$ in finite time over $(0, b)$, for then we would have $V\left(t_{1}\right)=z$ for some $0<t_{1}<b$ and thus $V^{\prime}\left(t_{1}\right)=0$ by (3.18), which would imply $V \equiv g \equiv z$. This is in contradiction with the fact that $V(0)=0$. Furthermore, $V^{\prime}$ cannot vanish on $(0, b)$. Indeed, if this was not true, and since $V^{\prime}(0)>0$, let $t_{0}>0$ be the first time where $V^{\prime}$ vanishes. Then, as a consequence of (3.18), we deduce that

$$
F\left(V\left(t_{0}\right)\right)=F(z) \text { for } 0<V\left(t_{0}\right)<z .
$$

This is impossible as $z \in \mathcal{Z}^{+}$. All these arguments show that $V$ is defined on $[0, \infty)$ and we set

$$
V_{z}=V \text {. }
$$

Note that $V_{z}^{\prime}>0$ and $0<V_{z}<z$ on $(0, \infty)$. Thus, $V_{z}(t)$ has a limit $l \in(0, z]$ as $t \rightarrow \infty$. We now show that $l=z$. Since $\lim _{t \rightarrow \infty} V_{z}^{\prime \prime}(t)=-f(l)$, we must then have $f(l)=0$. We also deduce that $\lim _{t \rightarrow \infty} V_{z}^{\prime}(t)=0$. Letting $t \rightarrow \infty$ in (3.18), we infer that

$$
F(l)=F(z) \text { for } 0<l \leq z,
$$

and again as $z \in \mathcal{Z}^{+}$, we deduce that $l=z$. For the uniqueness of $V_{z}$, we assume $V_{1}$ another solution of (1.3). Similar computations as above lead to

$$
\frac{1}{2}\left(V_{1}^{\prime}(t)\right)^{2}+F\left(V_{1}(t)\right)=c, \forall t \in[0, \infty) .
$$

Taking the limit as $t \rightarrow \infty$ in the above equality, we get $c=F(z)$ and hence $V_{1}^{\prime}(0)=$ $\sqrt{2 F(z)}$. Consequently, $V_{1} \equiv V_{z}$.

We now show that $\bar{u}$ given by (3.16) is a supersolution of (1.2). We first remark that

$$
\bar{u}=\min \left\{u^{+}, u^{-}\right\}
$$


where

$$
u^{+}=V_{z}\left(d^{+}\right) \text {and } u^{-}=V_{z}\left(d^{-}\right)
$$

and $d^{ \pm}=d^{ \pm}(x)$ be the distance measured from the point $x$ to the lines

$$
\left\{\left(r \cos \theta_{0}, \pm r \sin \theta_{0}\right): r \in \mathbb{R}\right\},
$$

respectively. We denote by $e^{ \pm}$the unitary vector directed orthogonally to the lines (3.19), respectively. By writing $u^{ \pm}(x)=V_{z}\left(d^{ \pm}(x)\right)=V_{z}\left(x \cdot e^{ \pm}\right)$and using the fact that $V_{z}$ is a solution of (1.3), it is easily seen that $u^{ \pm}$are both classical supersolutions of the differential equation of (1.2). We also observe that $\bar{u}=0$ on $\partial \Omega$ since $V_{z}(0)=0$. From all what precedes, we finally deduce (see again $[10,13,14]$ ) that $\bar{u}=\min \left\{u^{+}, u^{-}\right\}$is a supersolution of (1.2).

\section{Proof of Theorem 1.3}

This final section is devoted to the proof of the main result. Our next proposition compares $\underline{u}_{\varepsilon}$ and $\bar{u}$ over $\Omega$ by using a sliding argument.

Proposition 4.1 For $\varepsilon$ satisfying (2.13), let $\underline{u}_{\varepsilon}$ be the subsolution of (1.2) obtained by Proposition 2.1, and let $\bar{u}$ be the supersolution of (1.2) obtained by Proposition 3.1. Then,

$$
\underline{u}_{\varepsilon} \leq \bar{u} \text { on } \bar{\Omega} \text {. }
$$

Proof By the symmetry of $\bar{u}$ over $\Omega$, it suffices to show that

$$
\underline{u}_{\varepsilon} \leq u^{+} \text {on } \bar{\Omega} \text {. }
$$

Being generated by $\underline{u}_{\varepsilon, R}^{y}, y \in \Omega_{R}$ [see (2.15)] where $R=R(\varepsilon)>0$ is sufficiently large (see Lemma 2.3), we may further simplify the analysis by only comparing $\underline{u}_{\varepsilon, R}^{y}$ and $u^{+}$over $\Omega$. We have

$$
\underline{u}_{\varepsilon, R}^{y}=0 \leq V_{z}\left(d^{+}\right)=u^{+} \text {on } \bar{\Omega} \backslash \overline{B_{R}(y)},
$$

so, to complete the proof, it remains to show that, for every $y \in \Omega_{R}$,

$$
u_{\varepsilon, R}^{y} \leq u^{+} \text {on } \overline{B_{R}(y)} \subset \Omega .
$$

Firstly, since $u_{\varepsilon, R}$ satisfies the second relation in (2.10), and as $u_{\varepsilon, R}^{y}$ is a translation of $u_{\varepsilon, R}$, then we have $u_{\varepsilon, R}^{y}<z$ on the compact set $\overline{B_{R}(y)}$. As a result, we get

$$
u_{\varepsilon, R}^{y} \leq z-\beta \text { on } \overline{B_{R}(y)},
$$

for a sufficiently small $\beta>0$. Secondly, let $R^{\prime}>0$ be large enough such that $V_{z}(d)>z-\beta$ in $\Omega_{R^{\prime}}$, and fix $y_{0}$ such that the ball $B_{R}\left(y_{0}\right) \subset \Omega_{R^{\prime}}$. Thus

$$
u_{\varepsilon, R}^{y_{0}}<u^{+} \text {on } B_{R}\left(y_{0}\right) .
$$

This inequality on a particular ball $B_{R}\left(y_{0}\right)$ can be proved on any ball $B_{R}(y), y \in \Omega_{R}$ by using a classical sliding argument. This shows (4.21).

We are now ready to present the proof of our theorem. 
Proof of Theorem 1.3 Let $\underline{u}_{\varepsilon}$ be the subsolution of (1.2) obtained by Proposition 2.1. From Proposition 4.1, we know that

$$
\underline{u}_{\varepsilon} \leq \bar{u} \text {. }
$$

Let

$$
\underline{u}=\sup \underline{u}_{\varepsilon}
$$

where $\varepsilon$ ranges in $\left(0, z_{1}\right)$ if $z=z_{1}$ or in $\left(0, \min \left\{\delta, \delta^{\prime}\right\}\right)$ [see (2.13)] if $z \neq z_{1}$. From (4.22), we deduce that $\underline{u}$ is finite and again a subsolution of (1.2) with

$$
\left\{\begin{array}{l}
\underline{u} \leq \bar{u} \text { in } \Omega, \\
\left.\underline{u}\right|_{\partial \Omega}=\left.\bar{u}\right|_{\partial \Omega}=0 .
\end{array}\right.
$$

Using sub- and supersolution method (see $[10,13]$ ), we get the existence of a solution $u$ of (1.2) satisfying:

$$
\underline{u} \leq u \leq \bar{u},
$$

hence

$$
0<u \leq V_{z}(d) \text { in } \Omega .
$$

To complete the proof, we need to show the convergence in (1.4). Indeed, for any $\varepsilon>0$ small enough, Proposition 2.1 ensures the existence of $R=R(\varepsilon)>0$ such that

$$
z-\underline{u}_{\varepsilon}<\varepsilon \text { for } d>R
$$

and consequently, thanks to (4.23) and the fact that $V_{z}<z$, we get

$$
V_{z}(d)-u<\varepsilon \text { for } d>R \text {. }
$$

This terminates the proof.

Acknowledgements The authors are indebted to the anonymous referees for their extensive comments and suggestions that enormously improved the final presentation of the paper. H. Ibrahim would like to thank E. Nasreddine for various stimulating discussions. This project has been funded with support from the Lebanese University.

\section{References}

1. Angenent, S.: Uniqueness of the solution of a semilinear boundary value problem. Math. Ann. 272(1), 129-138 (1985)

2. Berestycki, H., Caffarelli, L., Nirenberg, L.: Further qualitative properties for elliptic equations in unbounded domains. Ann. Scuola Norm. Sup. Pisa Cl. Sci. (4) 25(1-2), 69-94 (1997)

3. Berestycki, H., Caffarelli, L., Nirenberg, L.: Monotonicity for elliptic equations in unbounded Lipschitz domains. Commun. Pure Appl. Math. 50, 1089-1111 (1997)

4. Berestycki, H., Caffarelli, L., Nirenberg, L.: Symmetry for elliptic equations in a half space. In: Boundary Value Problems for Partial Differential Equations and Applications, RMA Research Notes in Applied Mathematics, Masson, Paris, vol. 29, pp. 27-42 (1993)

5. Berestycki, H., Nirenberg, L.: On the method of moving planes and the sliding method. Bol. Soc. Bras. Mat. 22, 1-37 (1991)

6. Berestycki, H., Nirenberg, L.: Some Qualitative Properties of Solutions of Semilinear Elliptic Equations in Cylindrical Domains, Analysis, et cetera, pp. 115-164. Academic Press, Boston (1990)

7. Berestycki, H., Nirenberg, L.: Travelling fronts in cylinders. Ann. Inst. H. Poincaré Anal. Non Linéaire 9(5), 497-572 (1992)

8. Busca, J., Efendiev, M., Zelik, S.: Classification of positive solutions of semilinear elliptic equations. C. R. Math. Acad. Sci. Paris 338(1), 7-11 (2004) 
9. Efendiev, M., Hamel, F.: Asymptotic bahavior of solutions of semilinear elliptic equations in unbounded domains: two approaches. Adv. Math. 228, 1237-1261 (2011)

10. Fukagai, N.: Existence and uniqueness of entire solutions of second order sublinear elliptic equations. Funkcial. Ekvac. 29(2), 151-165 (1986)

11. Gidas, B., Ni, W., Nirenberg, L.: Symmetry and related properties via the maximum principle. Commun. Math. Phys. 68, 209-243 (1979)

12. Ibrahim, H., Nasreddine, E.: Existence of semilinear elliptic equations with prescribed limiting behaviour. Math. Methods Appl. Sci. 39(14), 4129-4138 (2016)

13. Noussair, E.: On the existence of solutions of nonlinear elliptic boundary value problems. J. Differ. Equ. 34(3), 482-495 (1979)

14. Volpert, A., Khudyaev, S.: Analysis in Classes of Discontinuous Functions and Equations of Mathematical Physics. Nijhoff, Dordrecht (1985)

Publisher's Note Springer Nature remains neutral with regard to jurisdictional claims in published maps and institutional affiliations. 